\title{
CARACTERIZACIÓN EPIDEMIOLÓGICA DE LA CRIPTOSPORIDIOSIS EN BOVINOS EN LA REGIÓN SABANA CENTRO (CUNDINAMARCA)
}

\section{EPIDEMIOLOGIC CHARACTERIZATION OF CRYPTOSPORIDIOSIS IN CATTLE IN THE CENTER SABANA REGION (CUNDINAMARCA)}

\begin{abstract}
Catalina Avendaño ${ }^{1}$, Álvaro Amaya ${ }^{2}$, Martín Bayona ${ }^{3}$
${ }^{1}$ Microbióloga Agrícola y Veterinaria Esp. Facultad de Medicina Veterinaria Universidad de Ciencias Aplicadas y Ambientales, U.D.C.A, Calle 222 No. 55-37, Bogotá D.C., Colombia, cavendano@udca.edu.co ${ }^{2}$ Estudiante octavo semestre Programa Medicina Veterinaria, Facultad Ciencias Pecuarias U.D.C.A, alamayam89@hotmail.com ${ }^{3}$ Bacteriólogo MSc. Facultad Medicina Humana Universidad de Ciencias Aplicadas y Ambientales, U.D.C.A, Calle 222 No.55-37, Bogotá D.C., Colombia, mabayona@udca.edu.co
\end{abstract}

Rev. U.D.C.A Act. \& Div. Cient. 13 (2): 109-116, 2010

\section{RESUMEN}

Para contribuir al conocimiento de la criptosporidiosis, comprender la prevalencia de Cryptosporidium spp. en terneros y asociar la presencia de ooquistes a diferentes factores ambientales, se realizó un estudio, en el cual, se recolectaron 151 muestras de heces de terneros, de hasta un mes de nacidos, en la región Sabana Centro (Cundinamarca). Para identificar los ooquistes de Cryptosporidium spp., se empleó la técnica de Ziehl Neelsen modificada. El 22\% de las muestras fue positivo y en el $83 \%$ de las fincas había, al menos, un animal infectado. El análisis estadístico mostró una asociación entre la consistencia de la materia fecal y la presencia de ooquistes de Cryptosporidium spp. ( $X^{2} 16,3 ; p$ $<0,01)$. La fuente de agua de las fincas reveló una asociación estadísticamente significativa, cuando se comparó con la presencia de ooquistes en las heces $\left(x^{2} 11,79\right.$; $\left.p<0,008\right)$, siendo un factor de riesgo el agua de acueducto; contrario a lo esperado, el agua tratada mostró tener un efecto protector. Así mismo, se consiguió una relación estadísticamente significativa entre el tipo de alojamiento y la presencia de ooquistes $\left(x^{2} 7,23 ; p<0,02\right)$, con una mayor probabilidad de hallar ooquistes en los animales que estaban en estaca que en aquellos que permanecían estabulados (OR > 4,03). Los resultados obtenidos confirman que la infección por Cryptosporidium spp. está ampliamente distribuida en los hatos lecheros de la zona geográfica de estudio.

Palabras clave: Cryptosporidium, terneros, heces, Ziehl Neelsen, diarrea.

\section{SUMMARY}

In order to contribute to the knowledge of cryptosporidiosis, the prevalence of Cryptosporidium spp. in calves and the association of the oocysts presence, related to different environmental factors was studies in 151 samples collected from faeces of calves up to one month old in the region Sabana Centro (Cundinamarca). To identify the Cryptosporidium spp. oocysts the Ziehl Neelsen technique was employed. $22 \%$ of the samples were positive; $83 \%$ of farms had at least one infected animal. Statistical analysis showed an association between the consistency of the stool and the presence of Cryptosporidium spp. oocysts ( $\mathrm{X}^{2} 16.3, p<0.01$ ); the water source of the farms showed a statistically significant influence when compared with the presence of oocysts in the feces $\left(\mathrm{X}^{2} 11.79, p<0.008\right)$, being a risk factor the aqueduct water, contrary to expectations, the farm treated water showed a protective effect. Likewise, we obtained a statistically significant relationship between the type of accommodation and the presence of oocysts $\left(\mathrm{X}^{2} 7.23, p\right.$ $<0.02$ ), with a greater likelihood of finding oocysts in animals that were in stake than those which remained in cowshed (OR> 4.03). The results confirm that Cryptosporidium spp. infection is widespread in dairy herds in the geographical study area.

Key words: Cryptosporidium, calves, feces, Ziehl Neelsen, diarrea. 


\section{INTRODUCCIÓN}

Cryptosporidium spp. es un parásito protozoo de distribución mundial, el cual, ha sido encontrado en vertebrados e invertebrados (Wielinga et al. 2008) y ha demostrado poseer potencial zoonótico (Brook et al. 2009) que ocasiona gastroenteritis severa en humanos y en otros animales, incluidos los porcinos (Johnson et al. 2008; Xiao et al. 2006). Alrededor del mundo, este parásito se ha constituido como una de las principales causas de diarrea en los humanos y uno de los principales protozoos que causa enfermedad, a través del agua (Hashimoto et al. 2006; Smith et al. 2007).

Cryptosporidium spp. pertenece al phylum Apicomplexa (= Sporozoa), cuyos miembros poseen un complejo apical; clase Sporozoae y se reproducen por ciclos sexuales y asexuales; subclase Coccidia, donde el ciclo de vida involucra merogonia, gametogonia y esporogonia; orden Euciccidiida (= Eucoccidiorida), en los que ocurre la esquizogonia; suborden Eimeriina (= Eimeriorina), en los cuales, la microgamia y la macrogamia se desarrollan de manera independiente; familia Cryptosporidiidae, cuyos miembros tiene cuatro esporozoitos desnudos dentro del ooquiste (Plutzer E Karanis, 2009).

Se han reconocido 22 especies de Cryptosporidium spp. (Fayer, 2010): cuatro en peces, una en anfibios, dos en reptiles, tres en aves y doce en mamíferos (Plutzer $\mathcal{E}$ Karanis, 2009). Algunas especies parecen estar restringidas a un solo hospedador mientras que otras no son hospedador específico (Thompson et al. 2008).

El estadio del parásito responsable de la transmisión de la enfermedad son los ooquistes (Fayer, 2010), los que son altamente resistentes al estrés ambiental y a los tratamientos, como la desinfección química. Esto se atribuye a su gruesa pared compuesta por una compleja barrera protectora, que le permite sobrevivir por largos periodos fuera del hospedador (Plutzer E Karanis, 2009). Los ooquistes son eliminados en grandes cantidades en las heces de los animales y de los humanos, parasitados durante la fase aguda de la infección, pudiendo mantenerse infectantes durante periodos prolongados. Esta circunstancia, unida a la baja dosis infectante (10-100 ooquistes), el elevado número de especies animales que actúan como reservorios del parásito y la ausencia de un tratamiento farmacológico eficaz, facilitan la difusión de la enfermedad (Casemore et al. 1997).

El principal reservorio es el ganado bovino, específicamente, los terneros, en los que la criptosporidiosis es responsable de una alta tasa de morbilidad neonatal (Broglia et al. 2008). Cryptosporidium spp. tiene un gran potencial de transmisión a través del agua de bebida, debido a que los ooquistes pueden penetrar las barreras físicas usadas en el tratamiento, además, de ser resistentes a los desinfectantes usados en este proceso, todo esto, ligado a la baja dosis infectante para los humanos y para los animales (Smith et al. 2007). La infección en los bovinos está altamente relacionada con la edad, donde las mayores prevalencias e intensidad de eliminación de ooquistes se ven en los terneros jóvenes (Brook et al. 2008).

Cryptosporidium spp. es un parásito reconocido como una de las principales causas de diarreas en terneros de hasta un mes de edad. La infección es responsable de una alta mortalidad (Klein et al. 2008); por ejemplo, Singh et al. (2006), en un estudio realizado en Punjab (India), encontraron una tasa de mortalidad del $35,2 \%$ y una tasa de fatalidad del $44,4 \%$. Su impacto se asocia, principalmente, con el daño del intestino y la baja conversión del alimento. Alrededor del mundo, en las explotaciones ganaderas, la principal fuente de contagio la constituyen los propios animales enfermos, que contaminan con sus heces la cama de la explotación. La prevalencia suele ser mayor en explotaciones, con un elevado número de animales y determinados factores, como el hacinamiento y las condiciones higiénicas deficientes, que se consideran factores de riesgo. Las anteriores consideraciones justifican que la presentación clínica de la enfermedad esté asociada con la época de partos, observándose un marcado incremento en la incidencia de nuevos casos al final de la misma, como consecuencia de la contaminación progresiva de la explotación, a lo largo de la paridera. Los animales adultos también desempeñan un papel importante en la transmisión, puesto que pueden actuar como portadores asintomáticos que eliminan un reducido número de ooquistes, aunque suficiente para infectar a los animales recién nacidos (Vergara E Quílez, 2004).

Un ternero con diarrea puede excretar $10^{7}$ ooquistes por gramo de heces y, así, puede producir billones de ooquistes durante una a dos semanas, en las que la infección está latente (O'Handley, 2007).

Por otra parte, la resistencia de los ooquistes a los tratamientos y a los desinfectantes utilizados rutinariamente para potabilizar el agua de bebida ha dado notoriedad a la criptosporidiosis en los últimos años, como enfermedad de transmisión hídrica, que hoy en día se considera como uno de los mecanismos de transmisión de la enfermedad al hombre, de mayor interés (Widme et al. 1996). De hecho, en los últimos años se han documentado un total de 39 brotes hídricos en Reino Unido, Estados Unidos, Canadá y Japón (Slifko, 2000), destacando el ocurrido en la ciudad norteamericana de Milwaukee en 1993, donde 400.000 personas resultaron afectadas: un $10 \%$ requirieron ingreso hospitalario y, aproximadamente, cien murieron (Mac Kenzie et al. 1994). 
En lo que se refiere a la influencia del clima existen diferentes corrientes; Kvác et al. (2006) indican que la presencia del parásito en los hatos es un constante hecho que se puede dar por la resistencia ambiental de ooquistes de Cryptosporidium spp. y la adición permanente al hato de nuevos terneros susceptibles. El proceso de transmisión es continua en las granjas lecheras, contrario a lo reportado por Trotz-Wiliams et al. (2007), quienes encontraron que los terneros nacidos en Canadá, durante los meses de verano, tenían una mayor probabilidad de eliminar Cryptosporidium spp., hallazgo diferente a lo reportado en estudios realizados en Nueva York y en otras ciudades con condiciones climáticas similares, cuyos descubrimientos indicaron que el invierno representa un riesgo para los terneros (Szonyi et al. 2010).

Los tratamientos para la criptosporidiosis son más paliativos que curativos, con excepción de nitazoxanida, la cual, ha mostrado eficacia en el tratamiento de pacientes inmunocompetentes, así como inmunocomprometidos, con excepción de los niños con VIH hospitalizados. El uso de calostro bovino hiperinmune ha demostrado una reducción de los síntomas clínicos de la enfermedad, así como de la deflación de la eliminación de ooquistes, en varias especies animales. De manera interesante, el calostro bovino no inmune también ha mostrado eficacia en personas inmunocomprometidas, lo que se puede deber a la presencia de algunos ácidos grasos insaturados (linoleico, oleico), que inhiben la adhesión de los esporozoitos al enterocito (Schmidt E Kuhlenschmidt, 2008).

Parece que Cryptosporidium paruum tiene una resistencia natural a quimioterapia y varios factores pueden contribuir a esta falta de eficacia, que incluyen: 1) la ubicación única del parásito en la célula hospedadora puede afectar la concentración del fármaco (transportado desde la célula hospedadora a través del parásito); 2) falta de objetivos específicos y las diferencias de objetivos ya sea molecular o estructuralmente; 3) diferencias en las vías bioquímica y 4) la existencia de proteínas de transporte o bomba de expulsión que transportan el medicamento fuera del parásito o dentro de la célula hospedadora (Mead, 2002).

En Sudamérica, no se conoce con exactitud la prevalencia de la criptosporidiosis, dado que los estudios epidemiológicos realizados son escasos, a pesar que el parásito ha sido identificado en todos los países en los que se investigó su presencia, mediante técnicas coprológicas (Vergara \& Quílez, 2004). Por ejemplo, Surumay \& Alfaro (2000) encontraron una prevalencia del $29,3 \%$ en la región occidental de Venezuela en animales que tenían entre 2 y 20 semanas de edad. En un estudio llevado a cabo en diferentes regiones de Argentina mostró una prevalencia del parásito del $29,6 \%$ en terneros de lecherías y 30,5\% en terneros de ganaderías de carne (Bellinzoni et al. 1990). En otro estudio en el Distrito de Magdalena (Argentina) hallaron un prevalencia del $17 \%$ en terneros menores de 30 días (Del Coco et al. 2008). Las mayores prevalencias de Cryptosporidium spp. han sido reportadas en terneros de dos semanas de edad (Brook et al. 2009; Trotz-Wiliams et al. 2007; Kvác et al. 2006).

En el departamento de Cundinamarca, Colombia, entre junio de 1996 y octubre de 1998, se realizó el primer estudio serológico de Cryptosporidium spp. en bovinos adultos, en el cual, se registró una seroprevalencia de 53,3\%, lo que indicó que la criptosporidiosis es endémica y representa un importante problema de salud pública en el país (Vergara et al. 2000). En el Valle de Ubaté y de Chiquinquirá, se halló una prevalencia del $22 \%$ en terneros de hasta 35 días (Avendaño et al. 2010).

Este trabajo buscó determinar la prevalencia de Cryptosporidium spp., así como contribuir al conocimiento de la criptosporidiosis en Colombia, mediante un estudio epidemiológico en la región Sabana Centro (Cundinamarca), en terneros lactantes en explotaciones de ganado vacuno.

\section{MATERIALES Y MÉTODOS}

Desde septiembre de 2009 hasta abril de 2010, se recolectaron y se procesaron 151 muestras de materia fecal de terneros menores de un mes, de hatos localizados en la región Sabana Centro de Cundinamarca. Las muestras fueron analizadas con la técnica de Ziehl Neelsen (ZN) modificado, como le reportaron Henricksen $\mathcal{E}$ Pohlenz (1981) y se consideraron positivas cuando se observó al menos un ooquiste del parásito (llustración 1a). Dada la baja especificidad y sensibilidad de ZN, las muestras fueron confirmadas con el uso de un kit comercial de inmunofluorescencia (IFA) (Ilustración 1b), debido a que la IFA es una herramienta útil para las muestras que tienen un poco número de ooquistes (Quílez et al. 1996). Las muestras fueron recolectadas mensualmente, en doce fincas ubicadas en los municipios de Tenjo, Cota, Chía, Tocancipá, Gachancipá y Cogua. Cada ternero fue muestreado una sola vez. Todos los animales muestreados tomaron calostro durante las primeras 24 horas de vida y consumían leche corriente.

En cada finca, se aplicó una encuesta al propietario, donde se registraron varios datos, como la fuente de agua, el tamaño del hato, el número de terneros, el tipo de alojamiento, la dieta, la edad del ternero y si el ternero había ingerido calostro. Adicionalmente, se solicitaron los antecedentes de pluviometría al Instituto de Hidrología, Meteorología y estudios ambientales de Colombia (IDEAM). 


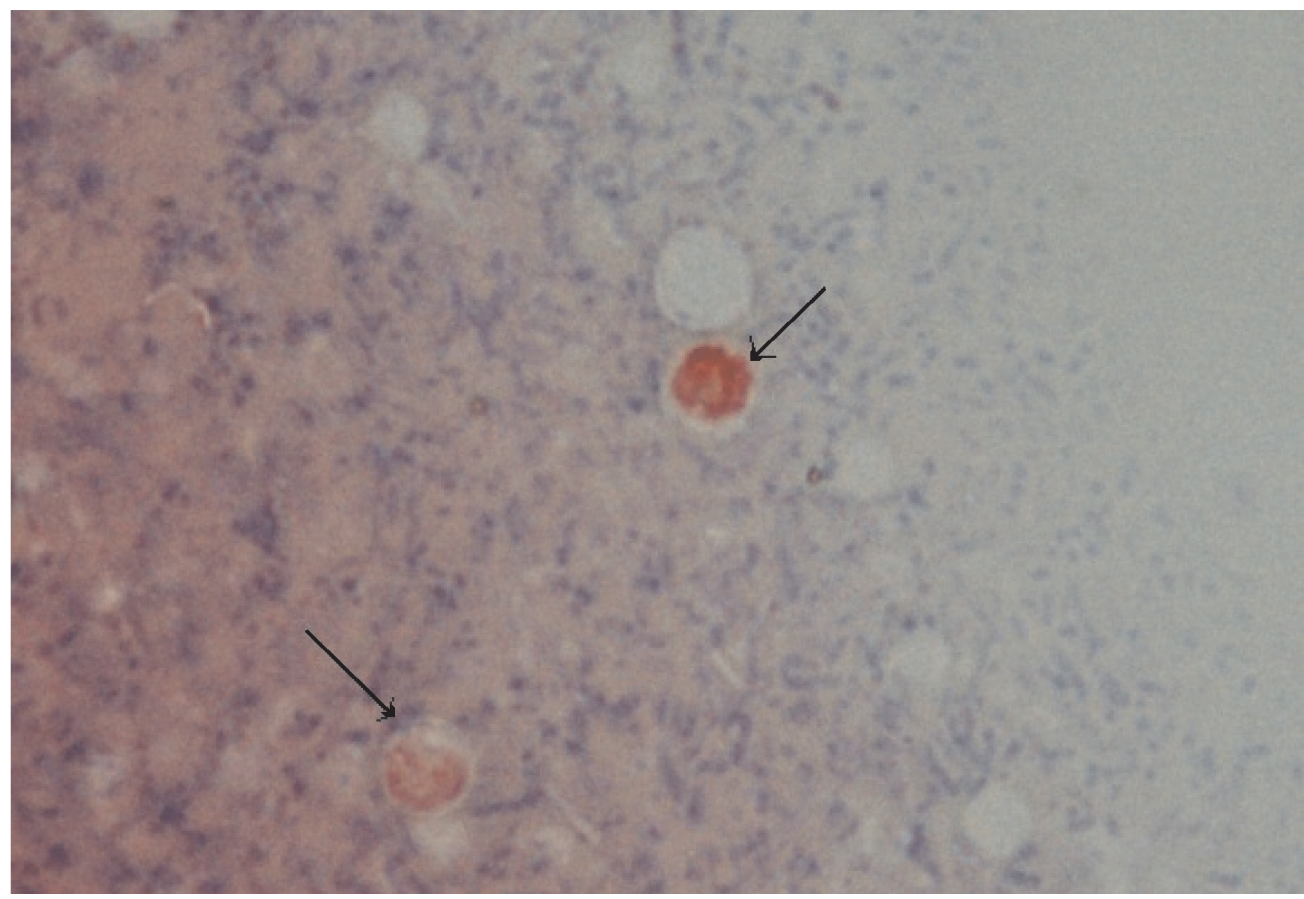

llustración 1a: Técnica Ziehl Neeelsen modificado. Ooquistes de Cryptosporidium spp. obtenidos de materia fecal de terneros menores de cinco semanas, en la Región Sabana Centro (Cundinamarca).

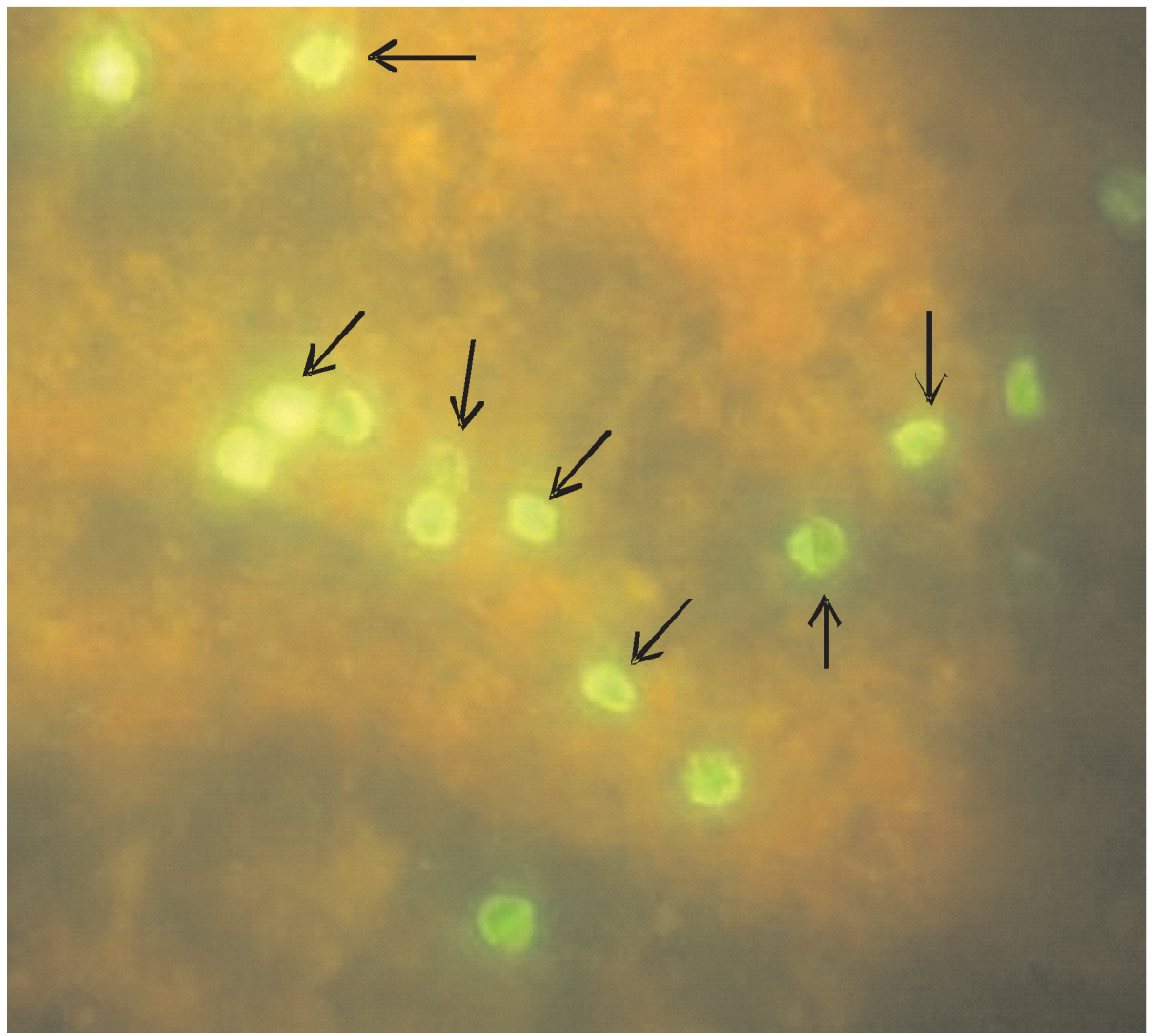

\|lustración $1 \mathrm{~b}$ : Técnica IFA. Ooquistes de Cryptosporidium spp. provenientes de materia fecal de terneros menores de cinco semanas, en la Región Sabana Centro (Cundinamarca). 
Para analizar la fuerza de asociación entre las variables estudiadas, se realizó la prueba de $X^{2}$ y se halló el $p$-valor; para rechazar la hipótesis nula, se tomó como valor de referencia un $p<0,05$. Cuando en los valores esperados fueron menores de cinco, se aplicó la prueba de Fisher. Para conocer la probabilidad que tenían los animales expuestos de presentar la enfermedad, se halló odds ratio (OR); las variables analizadas en este estudio fueron:

- Edad

- Consistencia de las heces

- Tipo de alojamiento

- Permanencia del ternero con la madre

- Tamaño del hato

- Número de terneros en el hato

- Fuente de agua de la explotación

- Volumen de precipitaciones

\section{RESULTADOS Y DISCUSIÓN}

De las 151 muestras en 33 (22\%), se observó al menos un ooquiste de Cryptosporidium spp. Los resultados obtenidos coinciden con los registrados por Avendaño et al. (2010) en el Valle de Ubaté y Chiquinquirá, en donde también se encontró una prevalencia del $22 \%$. Los datos concuerdan con los hallazgos realizados por Surumay \& Alfaro (2000), quienes indican una prevalencia del 29,3\%, en terneros de Venezuela. En Argentina, un estudio similar reportó una prevalencia del 17\% (Del Coco et al. 2008), cifra también cercana a la lograda en este esta investigación. En el $83 \%$ de los hatos muestreados, se halló al menos un animal positivo. Ya que en este estudio no se realizó la caracterización molecular del parásito y dadas las prevalencias comprobadas, los animales enfermos deben ser aislados y manipulados con precaución para evitar la transmisión zoonótica, así como el contagio a los otros animales del hato, ya que, de acuerdo a lo reportado por Santin et al. (2004), estos terneros pueden estar eliminando ooquistes de C. parvum, los cuales, se consideran patógenos para los humanos. Con esta investigación, se continua demostrando que Cryptosporidium spp. es un parásito que está ampliamente distribuido en nuestro país.

El 13\% de los 151 animales muestreados tenían una semana de edad; $22 \%$, estaba en el grupo de los de dos semanas; un $20 \%$, tres semanas; $21 \%$ de terneros cuatro semanas y, un $24 \%$, cinco semanas de nacidos. En todos los grupos de edades, se evidenció, al menos, un animal positivo, lo que confirma que los animales menores de treinta días son susceptibles al parásito (llustración 2). No se encontró una asociación estadísticamente significativa al comparar los grupos de edades trabajados con la presencia de ooquistes en la muestra examinadas, con excepción de aquellos terneros que tenían cuatro semanas ( $p<0,03$, OR 0,30$)$, los cuales, adicionalmente, presentaron menor positividad (9\%), reflejando ser esta edad en la que el ternero, al parecer, se encuentra protegido. En los terneros de dos semanas de edad hubo una mayor probabilidad de hallar el parásito, ya que en el $30 \%$ de ellos, se observaron ooquistes. Este periodo coincide con el periodo de prepotencia, que ha sido reportado entre 2 y 7 días (Fayer, 2008). Al comparar la edad en la que el ternero era separado de la madre ( 2 días, 3 días, 4 días), no hubo una relación estadísticamente significativa entre la edad de destete y la presencia de ooquistes $(p>0,2)$, dato que concuerda con la investigación de Trotz-Williams et al. (2007) quienes no encontraron una relación entre el tiempo que permanecen los terneros con la madre y la eliminación de Cryptosporidium spp., dato que contrasta con lo reportado por Silverlás et al. (2009), quienes indican que a mayor tiempo de permanencia del ternero con la madre se aumenta el OR, convirtiéndose en un factor de protección. Similar a lo reportado por Maddox et al. (2006), el tamaño del hato de las fincas muestreadas, durante el presente estudio, no tuvo relación estadísticamente significativa con la presencia de ooquistes en las heces de los terneros $(p>0,14)$. El hecho de haber encontrado una mayor positividad en los animales de dos semanas de vida y no haber hallado relación entre el tiempo de permanencia del ternero con la madre y la observación de ooquistes en las heces, puede estar indicando que en la región estudiada los animales no se están infectando al nacimiento sino cuando ingresan a los terneriles.

De los 33 animales positivos, las heces de 16 eran líquidas, mientras que las 17 restantes presentaban una consistencia pastosa. Del total de animales muestreados, 34 tenían heces líquidas, en tanto que 117 eran pastosas, encontrándose un relación estadísticamente significativa entre la observación de ooquistes y la presencia de diarrea $\left(x^{2} 16,3 ; p<0,0001\right)$. Este estudio mostró que en Sabana Centro de Cundinamarca existen cinco veces más probabilidades de hallar ooquistes en las heces líquidas (OR 5,2). Los datos obtenidos coinciden con otros investigadores, quienes afirman que la criptosporidiosis puede estar asociada con diarrea, especialmente en animales menores de un mes de edad (Silverlás et al. 2009; TrotzWiliams et al. 2007), aunque también puede estar ausente aun en animales fuertemente infectados (Lassen et al. 2009). Los animales sin diarrea, que también eliminaron ooquistes, se constituyen en un mayor riesgo para el trabajador y para los demás humanos, así como para los otros terneros, dado que pronto contaminarán los potreros y las fuentes de agua y al no presentar signos estos serán manipulados sin precaución, lo que representa un problema mayor para la salud pública y 


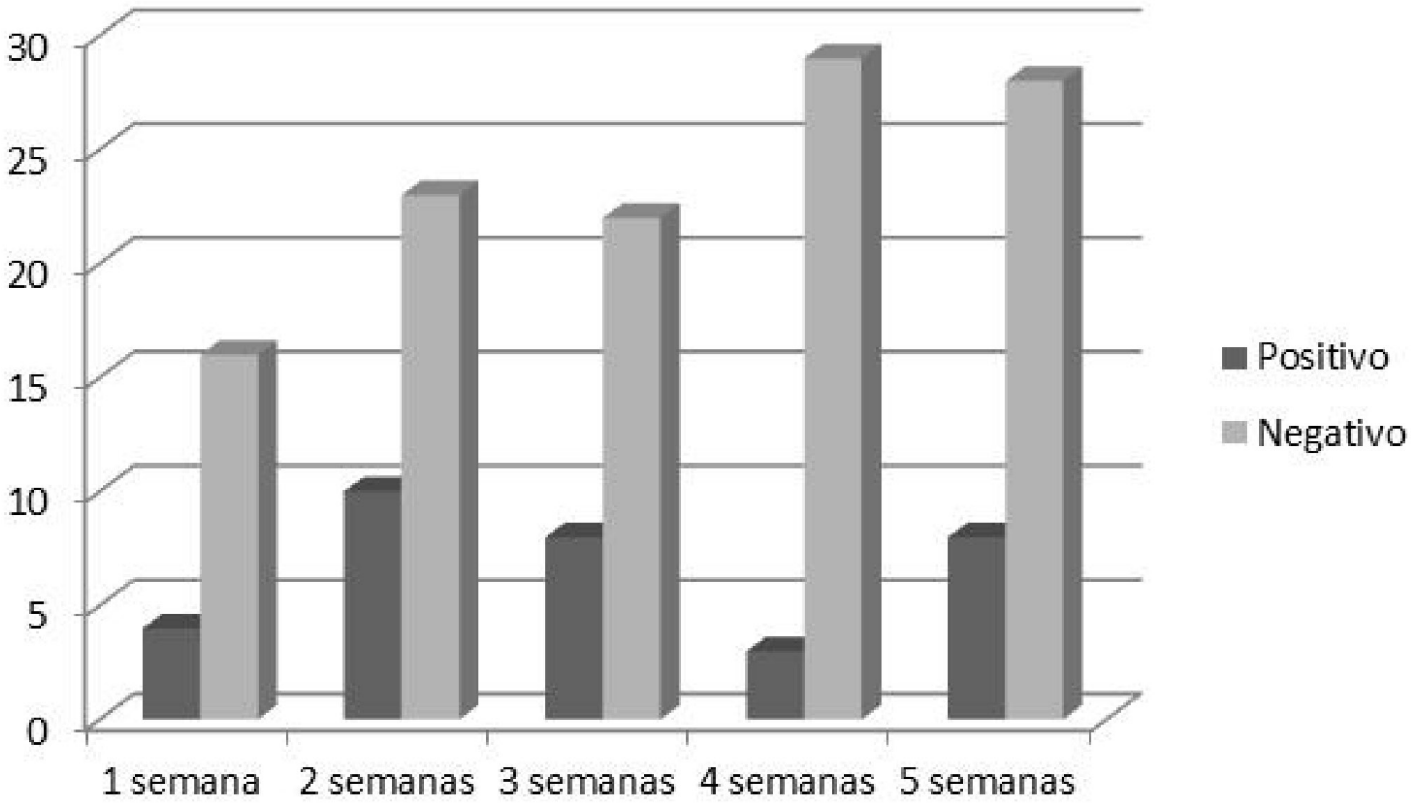

llustración 2: Proporción de animales positivos a Cryptosporidium spp. según la edad, en terneros menores de cinco semanas de edad, en la Región Sabana Centro (Cundinamarca).

para la salud del hato, que el generado por los animales que presentan diarrea.

Si bien es cierto que por la edad algunos terneros no ingerían agua directamente, los comederos eran lavados y no se secaban antes de entrar en contacto con la comida de los animales. De los 33 terneros positivos, 13 estaban en contacto con agua de acueducto, diez con agua tratada en las fincas, dos con agua proveniente del reservorio y ocho con agua de pozo profundo. Cryptosporidium spp. es un parásito altamente resistente a los sistemas de cloración y de tratamientos de agua (Quílez et al. 2008); sin embargo, contrario a lo esperado, la fuente de agua de las fincas mostró una asociación estadísticamente significativa, cuando se compararon las fincas, cuya fuente de agua provenía del acueducto con las demás fuentes ( $p<0,005)$. En los terneros alojados en fincas que usaban agua de acueducto de la vereda hubo tres veces más de probabilidades $(\mathrm{OR}>3,18)$ de encontrar ooquistes de Cryptosporidium spp., que en los que tenían otras fuentes de agua.

De los 151 animales muestreados, 92 estaba en estaca, 45 estabulados y 14 semiestabulados. De los 33 animales positivos, 26 estaban en estaca, cuatro estabulados y tres semiestabulados. Este estudio arrojó como resultado una relación estadísticamente significativa, cuando se compararon los animales positivos que estaban en estaca con los demás tipos de alojamientos ( $\left.x^{2} 5,65 ; p<0,01\right)$, existiendo una mayor probabilidad de encontrar ooquiste de Cryptosporidium spp. en los animales que estaban en estaca que en aquellos que permanecían estabulados (OR > 2,92), convirtiéndose en un factor de protección la estabulación (OR $<0,25)$, lo que coincide con lo reportado por Trotz-Wiliams et al. (2008), quienes hallaron que el uso de piso de concreto es un factor de protección. Esto puede estar relacionado con la fuente de agua, ya que los animales que se encontraban estabulados o semiestabulados no estaban en contacto con agua de acueducto, la que reveló ser un factor de riesgo para los terneros. La relación entre el número de terneros en la finca y la presencia de ooquistes en la muestra expuso una relación estadísticamente significativa $\left(x^{2} 8,47 ; p<0,003\right)$ existiendo más animales positivos en aquellas fincas que tenían menos de 15 terneros. Estas fincas tenían los terneros en estaca, mientras que las que poseían más de 15 , con excepción de una, los tenían estabulados o semiestabulados, lo que mostró ser un factor de protección. Adicionalmente, las fincas que tenían menos de 15 terneros utilizaban agua de acueducto de la vereda, lo que indicó ser un factor de riesgo, lo cual, puede estar dado por lo reportado por Smith et al. (2007), quienes indicaron que los ooquistes presentes en las heces pueden contaminar el agua directa o indirectamente y la disposición de los desechos humanos y de animales representa un ítem importante en esta enfermedad. 
Al analizar los datos suministrados por el IDEAM, se observó una relación entre la disminución de las precipitaciones mensuales y el hallazgo de ooquistes en las heces $(p<0,05)$; los meses en los que hubo menos animales positivos fueron los meses secos (enero, febrero y marzo), siendo estos un factor de protección para los terneros. Este hecho puede estar dado porque al no haber lluvias las fuentes de agua, de las cuales proviene para los acueductos y los pozos profundos, no sufre movimiento.

Agradecimientos. Los autores agradecen al Instituto Colombiano Agropecuario (ICA) y a los Laboratorios Vecol por el préstamo del microscopio de fluorescencia y a los propietarios de las fincas trabajadas durante la investigación. Conflicto de intereses: Los autores del presente escrito declaran que no existe conflicto de intereses que ponga en riesgo la validez de los resultados. Financiación: Este estudio fue financiado por el fondo de investigación de la Universidad de Ciencias Aplicadas y Ambientales U.D.C.A.

\section{BIBLIOGRAFÍA}

1. AVENDAÑO, C.; QUÍLEZ, J.; SÁNCHEZ, C. 2010. Prevalencia de Cryptosporidium en terneros en el Valle de Ubaté - Chiquinquirá (Colombia). Rev U.D.C.A Act E Div. Cient. 13(1):41-48.

2. BELLINZONI, R.; BLACKHALL, J.; TERZOLO, H.; MOREIRA, A.; AUZA, N.; MATTION, N.; MICHEO, G.; LA TORRE, J.; SCODELLER, E. 1990. Microbiology of diarrhoea in young beef and dairy calves in Argentina. Rev. Argent. Microbiol. 22(3):130-136.

3. BROGLIA, A.; RECKINGER, S.; CACCIÓ, S.; NÖCKLER, K. 2008. Distribution of Cryptosporidium parvum subtypes in calves in Germany. Vet. Parasitol. (Paises Bajos). 154(1-2):8-13.

4. BROOK, E.; ANTHONY, H.; FRENCH, N.; CHRISTLEY, R. 2009. Molecular epidemiology of Cryptosporidium subtypes in cattle in England. Vet. J. 179(3):378-382.

5. BROOK, E.; HART, C.; FRENCH, N.; CHRISTLEY, R. 2008. Prevalence and risk factors for Cryptosporidium spp. infection in young calves. Vet. Parasitol. 152(1-2):46-52.

6. CASEMORE, D.; WRIGHT, S.; COOP, R. 1997. Cryptosporidiosis - human and animal epidemiology. En: Fayer, R. Ed. Cryptosporidium and cryptosporidiosis. CRC Press, Boca Ratón, Florida. p.65-92.
7. DEL COCO, V.F.; CÓRDOBA, M.A.; BASUALDO, J.A. 2008. Cryptosporidium infection in calves from a rural area of Buenos Aires, Argentina. Vet Parasitol. 158(1-2):31-35.

8. FAYER, R. 2010, Taxonomy and species delimitation in Cryptosporidium. Exp. Parasitol. (Estados Unidos). 124(1):90-97.

9. FAYER, R. 2008. General biology. En: Fayer, R. Ed. Cryptosporidium and cryptosporidiosis. Second edition. CRC Press, Boca Ratón, Florida. p.1-42.

10. HASHIMOTO, A.; SUGIMOTO, H.; MORITA, S.; HIRATA, T. 2006. Genotyping of single Cryptosporidium oocysts in sewage by semi-nested PCR and direct sequencing. Water Res. (Inglaterra). 40(13):2527-2532.

11. HENRICKSEN, S.; POHLENZ, J. 1981. Staining of Cryptosporidium by a Modified Ziehl-Neelsen technique. Acta Vet Scand. (Inglaterra) 22(3-4):594-596.

12. JOHNSON, J.; BUDDLE, R.; REID, S.; ARMSON, A.; RYAN, U. 2008. Prevalence of Cryptosporidium genotypes in pre and post-weaned pigs in Australia. Exp. Parasitol. 119(3):418-421.

13. KLEIN, P.; KLEINOVÁ, T.; VOLEK, Z.; SIMワNEK, J. 2008. Effect of Cryptosporidium parvum infection on the absorptive capacity and paracellular permeability of the small intestine in neonatal calves. Vet Parasitol. 152(1-2):53-59.

14. KVÁC, M.; KOUBAET, M.; VÍTOVEC, J. 2006. Age-related and housing-dependence of Cryptosporidium infection of calves from dairy and beef herds in South Bohemia, Czech Republic. Vet. Parasitol. 137(3-4):202-209.

15. LASSEN, B.; VILTROP, A.; RAAPERI, K.; JÄRVIS, T. 2009. Eimeria and Cryptosporidium in Estonian dairy farms in regard to age, species, and diarrhea. Vet. Parasitol. 166(3-4):212-219.

16. MADDOX, C.; LANGKJ/ER, R.; ENEMARK, H.; VIGRE, H. 2006. Cryptosporidium and Giardia in different age groups of Danish cattle and pigs-Occurrence and management associated risk factors. Vet. Parasitol. 141(1-2):48-59.

17. MAC KENZIE, W.; HOXIE, N.; PROCTOR, M.; GRADUS, M.; BLAIR, K.; PETERSON, D.; KAZMIERCZAK, J.; ADDISS, D.; FOX, K.; ROSE, J. 1994. A massive outbreak in Milwaukee of Cryptosporidium infection transmitted through the public water. N. Engl J Med. 331(3):161-167. 
18. MEAD, J. 2002. Cryptosporidiosis and the challenges of chemotherapy. Drug Resist. Updat. (Escocia). 5(1):4757.

19. O'HANDLEY, R. 2007. Cryptosporidium parvum infection in cattle: are current perceptions accurate? Trends Parasitol. (Inglaterra). 23(10):477-480.

20. PLUTZER, J.; KARANIS, P. 2009, Genetic polymorphism in Cryptosporidium species: an update. Vet. Parasitol. 165(3-4):187-199.

21. QUÍLEZ, J.; SÁNCHEZ-ACEDO, C.; CLAVEL, A.; DEL CACHO, E.; LÓPEZ-BERNAD, F. 1996. Comparison of an acid-fast stain and a monoclonal antibody-based immunofluorescence reagent for the detection of Cryptosporidium oocysts in faecal specimens from cattle and pigs. Vet. Parasitol. 67(1-2):75-81.

22. QUÍLEZ, J.; TORRES, E.; CHALMERS, R.; ROBINSON, G.; DEL CACHO, E.; SÁNCHEZ-ACEDO, C. 2008. Cryptosporidium species and subtype analysis from dairy calves in Spain. Parasitol. (Inglaterra). 135(14):16131620.

23. SANTIN, M.; TROUT, J.; XIAO, L.; ZHOU, L.; GREINER, E.; FAYER, R. 2004. Prevalence and age-related variation of Cryptosporidium species and genotypes in dairy calves. Vet. Parasitol. 122(2):103-117.

24. SCHMIDT, J.; KUHLENSCHMIDT, M. 2008. Microbial adhesion of Cryptosporidium parvum: identification of a colostrum-derived inhibitory lipid. Mol. Biochem. Parasitol. (Paises Bajos). 162(1):32-39.

25. SILVERLÁS, C.; EMANUELSON, U.; DE VERDIER, K.; BJÖRKMAN, C. 2009. Prevalence and associated management factors of Cryptosporidium shedding in 50 Swedish dairy herds. Prev. Vet. Med. (Paises Bajos). 90(3-4):242-253.

26. SLIFKO, S. 2000. Emerging parasite zoonoses associated with water and food. Int. J. Parasitol. (Inglaterra). 30(12-13):1379-1393.

27. SINGH, B.; SHARMA, R.; KUMAR, H.; BANGA, H.; AULAKH, R.; GILL, J.; SHARMA, J. 2006. Prevalence of Cryptosporidium parvum infection in Punjab (India) and its association with diarrhea in neonatal dairy calves. Vet. Parasitol. 31;140(1-2):162-165.

28. SMITH, H.; CACCIO, S.; COOK, N.; NICHOLS, R.; TAIT, A. 2007. Cryptosporidium and Giardia as foodborne zoonoses. Vet. Parasitol. 149(1-2):29-40.
29. SURUMAY, Q.; ALFARO, C. 2000. Cryptosporidium spp. in farms in the eastern region of Venezuela. Invest. Clin. (Venezuela). 41(4):245-250.

30. SZONYI, B.; BORDONARO, R.; WADE, S.; MOHAMMED, H. 2010. Seasonal variation in the prevalence and molecular epidemiology of Cryptosporidium infection in dairy cattle in the New York City Watershed. Parasitol Res. (Alemania). 107(2):317-325.

31. THOMPSON, R.; PALMER, C.; O'HANDLEY, R. 2008. The public health and clinical significance of Giardia and Cryptosporidium in domestic animals. Vet. J. (Inglaterra). 177(1):18-25.

32. TROTZ-WILLIAMS, L.; MARTIN, M.; LESLIE, K.; DUFFIELD, T.; NYDAM, D.; PEREGRINE, V. 2008. Association between management practices and within-herd prevalence of Cryptosporidium parvum shedding on dairy farms in southern Ontario. Prev. Vet. Med. 83(1):11-23.

33. TROTZ-WILLIAMS, L.; WAYNE, S.; LESLIE, K.; DUFFIELD, T.; NYDAM, D.; PEREGRINE, A. 2007. Calf-level risk factors for neonatal diarrhea and shedding of Cryptosporidium parvum in Ontario dairy calves. Prev. Vet. Med. 82(1-2):12-28.

34. VERGARA, C.; QUÍLEZ, J. 2004. Criptosporidiosis: una zoonosis parasitaria. MVZ-Córdoba. (Colombia). 9(1):363-372.

35. VERGARA, C.; SANTOS, S.; SANTOS, F.; ARES, M. 2000. La criptosporidiosis en la región andina de Colombia: seroprevalencia y reconocimiento de antígenos. Rev. Panam. Salud Pública. (Estados Unidos). 8(6):373-379.

36. WIDME, G.; CARRAWAY, M.; TZIPORI, S. 1996. Waterborne Cryptosporidium: A perspective from the USA. Parasitol. Today. (Inglaterra). 12(7):286-290.

37. WIELINGA, P.; DE VRIES, A.; VAN DER GOOT, T.; MANK, T.; MARS, M.; KORTBEEK, L.; VAN DER GIESSEN, J. 2008. Molecular epidemiology of Cryptosporidium in humans and cattle in The Netherlands. Internal. J. Parasitol. (Estados Unidos). 38(7):809-817.

38. XIAO, L.; MOORE, J.; UKOH, U.; GATEI, W.; LOWERY, C.; MURPHY, T.; DOOLEY, J.; MILLAR, B.; ROONEY, P.; RAO, J. 2006. Prevalence and identity of Cryptosporidium spp. in pig slurry. Appl. Environ. Microbiol. (Estados Unidos). 72(6):4461-4463.

Recibido: Junio 16 de 2010

Aceptado: Octubre 4 de 2010 